\title{
INFLUÊNCIA DA CONCENTRAÇÃO DE SAL NA ADSORÇÃO DE TENSOATIVOS IÔNICOS EM ARENITOS
}

\author{
F. D. S. CURBELO ${ }^{1}$, A. I. C. GARNICA ${ }^{1}$, G. Z. CLERICUZI ${ }^{1}$, R. S. CARDOSO ${ }^{1}$, J. C. O. \\ FREITAS $^{2}$ e D. M. A. MELO ${ }^{2}$ \\ ${ }^{1}$ Universidade Federal da Paraíba, Departamento de Engenharia Química. \\ ${ }^{2}$ Universidade Federal do Rio Grande do Norte, Instituto de Química \\ e-mail para contato: fabioladias@yahoo.com
}

\begin{abstract}
RESUMO - Dentre os métodos químicos utilizados para recuperação avançada de petróleo tem-se a Injeção de solução de tensoativos, que é um processo em que se pressupõe uma certa interação química entre o fluido injetado e os fluidos do reservatório. Entretanto, na injeção de tensoativos, inevitavelmente, ocorre a perda de tensoativo por adsorção nas rochas, podendo reduzir a eficiência do método utilizado. As elevadas concentrações salinas presentes nas bacias petrolíferas devem ser levadas em consideração durante a aplicação deste método. Neste trabalho, foram avaliadas as influências dos sais $\mathrm{KCl}$ e NaCl na adsorção de um tensoativo aniônico em arenito, nas concentrações de $2,0 \% \mathrm{KCl}$ e 1,$0 ; 2,0$ e 4,0\% $\mathrm{NaCl}$, em peso, e obtidas as isotermas de adsorção para cada um dos sais e suas respectivas concentrações. Para o $\mathrm{NaCl}$, as concentrações estudadas apresentaram semelhanças nas isotermas de adsorção e a concentração de sal influenciou na quantidade de tensoativo adsorvida. As avaliações do comportamento térmico das amostras de arenito puro e arenito adsorvido com sabão base foram verificados pelas análises de TG/DTG. Comparando os sais, verifica-se que a quantidade de tensoativo adsorvida foi menor com o $\mathrm{KCl}$, provavelmente, devido ao seu maior raio atômico. Deste estudo, conclui-se que o fator de recuperação de óleo é afetado, significativamente, pelas altas concentrações de sal, sendo necessário uma quantidade maior de tensoativo para se obter o mesmo fator de recuperação de petróleo.
\end{abstract}

\section{INTRODUÇÃ̃o}

Apesar da constante busca atual por energias alternativas que venham a substituir o petróleo, este ainda é a principal fonte de energia do mundo moderno. Com isso, os métodos de recuperação avançada (EOR) têm atraído uma atenção significativa para a produção de petróleo. Os processos químicos de recuperação avançada de petróleo são aqueles em que se pressupõe uma certa interação química entre o fluido injetado e os fluidos do reservatório e podem ser a injeção de solução alcalina, solução de tensoativos, microemulsão e/ou polímeros.

Os tensoativos, devido à sua natureza anfifílica, têm uma tendência natural de serem adsorvidos nas interfaces ou superfícies de forma convenientemente orientadas, reduzindo a tensão interfacial. Este fenômeno é responsável pela maioria de suas características e propriedades. Além de para reduzir a tensão interfacial, os tensoativos também são utilizados 
para mudar a molhabilidade da rocha e controlar a mobilidade dos fluidos para aumentar a quantidade de óleo recuperado dos reservatórios previamente tratados com métodos convencionais de recuperação, como a injeção de água (ShamsiJazeyi e. a.l, 2014).

Apesar do significante potencial que a injeção de tensoativos oferece como um processo químico de recuperação avançada de petróleo, ainda existe desafios relacionados à viabilidade econômica da aplicação deste método em muitos reservatórios. Um dos principais desafios é minimizar a perda de tensoativos devido à adsorção deles nas formações rochosa.

A adsorção a partir de soluções aquosas diluídas sobre superfícies sólidas (paredes, sólidos depositados, partículas em suspensão) envolve, especificamente, uma interação química ou física entre o adsorvato e o adsorvente. A interação química se refere a processos de troca iônica em que os contra-íons do substrato são substituídos pelos íons dos tensoativos de carga similar, formando ligações entre as moléculas do adsorvente (substrato) e do adsorvato (tensoativo) e interações de emparelhamento de íons, nas quais os íons do tensoativo são adsorvidos sobre os sítios opostamente carregados não ocupados pelos contra-íons. $\mathrm{O}$ conhecimento das características físico-químicas dos tensoativos e de seu comportamento em solução direciona a escolha do tipo e quantidade de tensoativo, ou de suas misturas, para alcançar o melhor desempenho em cada uma de suas aplicações.

As perdas te tensoativos por adsorção e precipitação provocam mudanças na interação entre o fluido deslocado (óleo) e o fluido injetado (solução tensoativo). As mudanças na adsorção são causadas, principalmente, devido à estrutura química dos tensoativos, propriedades da rocha, composição dos fluidos de reservatório e condições da solução injetada, como: salinidade, pH e temperatura (Somasundaran e Zhang, 2006).

Neste estudo, testes de adsorção estática, realizados em banho finito, foram realizados para verificar a influência da concentração dos sais $\mathrm{KCl}$ e $\mathrm{NaCl}$ e demonstrar seus efeitos na recuperação de petróleo.

\section{MATERIAIS E MÉTODOS}

\subsection{Materiais}

Os materiais utilizados durante os procedimentos experimentais foram o tensoativo aniônico sabão base (SB), gentilmente fornecido pela Gessy Lever, e os sais $\mathrm{KCl}$ e $\mathrm{NaCl}$, de grau analítico (P.A.).

\subsection{Concentração micelar crítica}

Nos processos químicos de recuperação avançada de petróleo, a concentração da solução de tensoativo a ser injetada nos poços de petróleo precisa estar acima do valor da c.m.c., pois seu maior efeito está na diminuição da tensão interfacial ou na promoção de estabilidade das espumas e, isso só pode ser alcançado, quando uma quantidade significante de micelas está presente (Schramm, 1992 e 2000). 
A c.m.c. é também de grande interesse, pois a concentrações acima desse valor, a adsorção na rocha-reservatório aumenta muito pouco, uma vez que, a c.m.c. representa a concentração de tensoativo na qual se está próximo da adsorção máxima. A c.m.c. do tensoativo foi determinada utilizando o equipamento SensaDyne Tensiometer, onde foram utilizados, aproximadamente, 30 $\mathrm{mL}$ de cada amostra à temperatura de $26^{\circ} \mathrm{C}$ (Curbelo, 2006). Neste trabalho, a concentração micelar crítica e o peso molecular do sabão base (SB) foram determinados por Curbelo (2006) e seus valores foram, respectivamente, $1,69 \mathrm{~g} / \mathrm{L}$ e $286,1 \mathrm{~g} / \mathrm{mol}$.

\subsection{Análise granulométrica}

A amostra de arenito foi adquirida no Laboratório de Weatherford, Texas, USA. Esta amostra, já calcinada, foi triturada e peneirada em uma série de peneiras da série tipo "tyler", com malhas de 14, 48 e 100 mesh, que correspondem as aberturas de 1,168 mm, 0,295 mm e 0,147 mm, respectivamente (Foust et al., 1982).

\subsection{Adsorção}

Os experimentos de adsorção de tensoativos na rocha, realizados em banho finito, foram conduzidos em erlenmeyers de $250 \mathrm{~mL}$, em temperatura ambiente. Em cada erlenmeyer, uma massa constante do adsorvente (arenito) igual a 0,1 g e concentrações diferentes de solução de tensoativos com sal foram adicionadas. Os erlenmeyers foram levados à incubadora, sob agitação constante, com a finalidade de atingir o equilíbrio. A mistura foi deixada em repouso durante o tempo suficiente para que a rocha decantasse e houvesse total separação da solução. Em seguida, a concentração do tensoativo em cada amostra foi determinada.

As capacidades de adsorção $(\Gamma)$ foram calculadas utilizando a equação (1):

$$
\Gamma=\frac{V\left(C_{0}-C_{e}\right)}{m}
$$

Em que: $\mathrm{C}_{0}$ é a concentração inicial de tensoativo $(\mathrm{g} / \mathrm{mL}) ; \mathrm{C}_{\mathrm{e}}$ é a concentração de tensoativo no equilíbrio $(\mathrm{g} / \mathrm{mL})$; V é o volume da solução $(\mathrm{mL})$; $\mathrm{m}$ é a massa do adsorvente $(\mathrm{g})$.

Nesta etapa, foram testadas a influência dos sais $\mathrm{KCl}$ e $\mathrm{NaCl}$, na adsorção do tensoativo SB na amostra de arenito. O estudo da adsorção feito com estes sais deve-se ao fato de que ambos estão presentes na rocha reservatório e, portanto, suas participações na recuperação de petróleo devem ser consideradas. Entretanto, o $\mathrm{NaCl}$ está em quantidade predominante em relação ao $\mathrm{KCl}$ e, assim, sua influência foi melhor estudada, avaliando, também, nas concentrações de $1 \%, 2 \%$ e $4 \%$ em peso. As concentrações de SB utilizadas variaram de 0,1 a 2,2 g/L.

\subsection{Métodos Espectroscópicos}

Estes métodos foram realizados com o objetivo de confirmar a adsorção do tensoativo na rocha e verificar a influência da concentração de sal na adsorção. 
Análise termogravimétrica (TG): A avaliação do comportamento térmico das amostras de arenito puro e arenito adsorvido com sabão base com adição de $4 \%$ em peso $\mathrm{NaCl}$ foi realizada em uma balança termogravimétrica SDT Q600 da TA Instruments. As análises foram realizadas da temperatura ambiente a $900^{\circ} \mathrm{C}$, com taxa de aquecimento de $10^{\circ} \mathrm{C} \mathrm{min}{ }^{-1}$, sob vazão de 100 $\mathrm{mL} \mathrm{min}^{-1}$ de $\mathrm{N}_{2}$, utilizando aproximadamente $30 \mathrm{mg}$ das amostras, com o objetivo de avaliar o grau de degradação do tensoativo a altas temperaturas e verificar, com isso, se o mesmo suporta as temperaturas elevadas em que os reservatórios de petróleo apresentam sem perder suas principais funções e características.

FTIR: Os espectros de absorção na região do infravermelho foram obtidos em um Espectrofotômetro de Infravermelho por Transformada de Fourier (FTIR) da Marca Shimadzu, Modelo IRPrestige-21, usando brometo de potássio ( $\mathrm{KBr}$ ) como agente dispersante. As pastilhas foram preparadas pela mistura de aproximadamente $0,7 \mathrm{mg}$ de amostra com uma quantidade suficiente de $\mathrm{KBr}$ para se atingir a concentração de $1 \%$ em massa da amostra. Foram analisadas seis amostras: tensoativo (sabão base); arenito puro; arenito adsorvido com sabão base sem sal; arenito adsorvido com sabão base e $1,0 \%$ em peso $\mathrm{NaCl}$; com 2,0 \% e $4 \%$ em peso $\mathrm{NaCl}$.

\section{RESULTADOS E DISCUSSÃO}

Os principais resultados obtidos deste estudo encontram-se a seguir.

\subsection{Análise granulométrica}

Para os estudos da adsorção do tensoativo no arenito, foi utilizada a amostra da faixa granulométrica de $-48+100 \#$, pois nesta faixa granulométrica foi obtida a maior fração mássica das partículas sólidas de arenito, 44,82\%. O diâmetro médio das partículas foi determinado pela média aritmética dos diâmetros da abertura das peneiras de 48 e 100 \#, conforme equação (2).

$$
d_{P}=\frac{d_{48 \#}+d_{100 \#}}{2}=\frac{295 \times 10^{-6} \mathrm{~m}+147 \times 10^{-6} \mathrm{~m}}{2}=221 \times 10^{-6} \mathrm{~m}
$$

\subsection{Adsorção}

Os resultados para avaliar a influência da concentração de sal na capacidade de adsorção do tensoativo pela rocha estão apresentados nas Figuras a seguir. A Figura 1 mostra a influência do $\mathrm{KCl}$. 


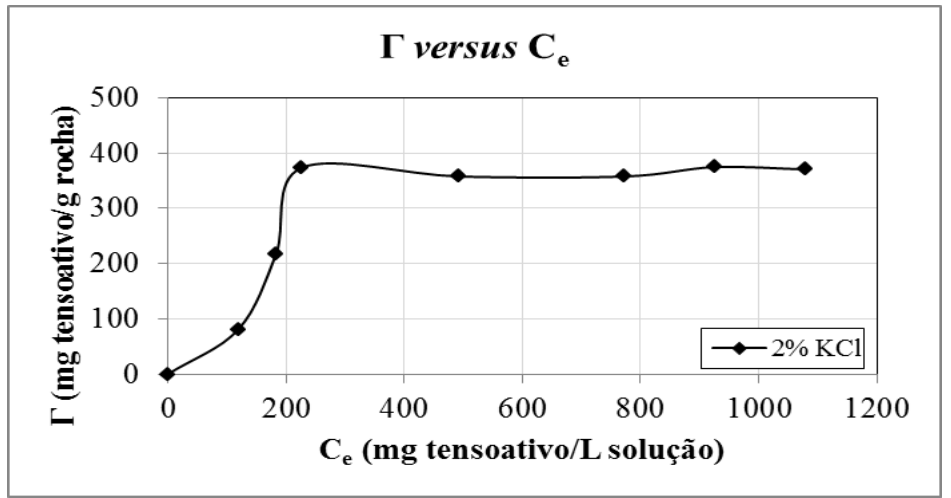

Figura 1 - Isoterma de adsorção com $2 \% \mathrm{KCl}$ em peso.

Observa-se, pela Figura 1, que a formação da monocamada, na isoterma de adsorção para a solução de tensoativo com $2 \% \mathrm{KCl}$ em peso, teve início a partir da concentração de equilíbrio $\left(\mathrm{C}_{\mathrm{e}}\right)$ aproximadamente igual a $226 \mathrm{mg} / \mathrm{L}$, com uma quantidade máxima adsorvida de tensoativo entre 350 e $400 \mathrm{mg}$ de tensoativo/g rocha.

A Figura 2 mostra as isotermas de adsorção para a solução de tensoativo com 1\%, 2\% e 4\% de $\mathrm{NaCl}$ em peso.

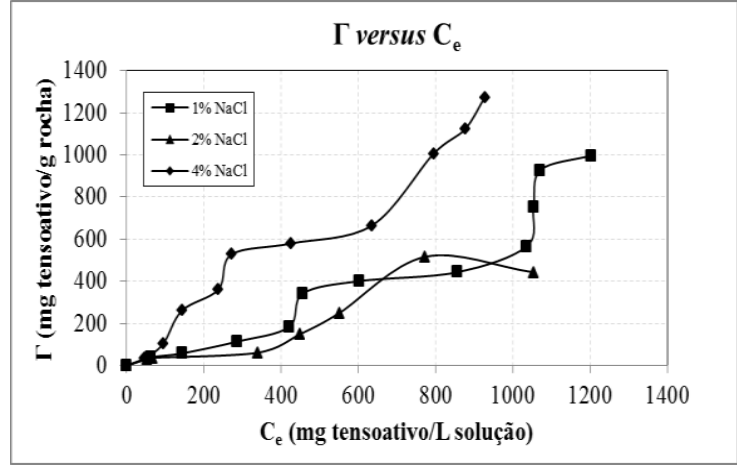

Figura 2 - Influência de 1\%, 2\% e 4\% em peso $\mathrm{NaCl}$ na adsorção do SB na rocha.

Observa-se, pela Figura 2, que para a concentração de $1 \%$ em peso $\mathrm{NaCl}$, a formação da monocamada se dá a partir da concentração de equilíbrio $\left(\mathrm{C}_{\mathrm{e}}\right)$ aproximadamente igual a 1070 $\mathrm{mg} / \mathrm{L}$, com uma quantidade máxima adsorvida de tensoativo entre 920 e $1000 \mathrm{mg}$ de tensoativo/g rocha, devido a interação entre as caudas apolares do tensoativo, em razão de estarem em concentrações próximas a da cmc. Para a concentração de $2 \%$ em peso $\mathrm{NaCl}$, o início da monocamada se deu a partir da concentração de equilíbrio $\left(\mathrm{C}_{\mathrm{e}}\right)$ aproximadamente igual a 770 $\mathrm{mg} / \mathrm{L}$, com uma quantidade máxima adsorvida de tensoativo entre 440 e $520 \mathrm{mg}$ de tensoativo/g rocha, que é menor do que para a $1 \% \mathrm{NaCl}$, devido a maior quantidade de sal em solução e, também, pelo fato do $\mathrm{NaCl}$ apresentar interações mais fortes com o adsorvente do o tensoativo. E para a concentração de $4 \%$ em peso $\mathrm{NaCl}$, observa-se uma tendência muito alta de adsorção do tensoativo, para as concentrações de tensoativo em solução estudadas, o que está em desacordo 
com a tendência do comportamento vistos em $1 \%$ e $2 \% \mathrm{NaCl}$. Entretanto, por se tratar de um tensoativo aniônico, sabe-se que a adsorção se resume a monocamada. A partir da concentração de $800 \mathrm{mg}$ tensoativo/ L solução, a adsorção acontece somente devido a interações hidrofóbicas cadeia-cadeia, combatidas pela repulsão eletrostática que se desenvolve quando a superfície começa a adquirir a mesma carga dos íons de tensoativo que estão sendo adsorvidos. A formação total da monocamada será atingida, provavelmente, quando a concentração de equilíbrio tiver próxima da cmc do tensoativo (1690 mg/L).

Verificando, de modo geral, a influência das concentrações de sal utilizadas (Figuras 2) observa-se que a influência do sal foi mais pronunciada na concentração de $4 \% \mathrm{NaCl}$, onde verifica-se que para uma mesma concentração de equilíbrio, por exemplo $600 \mathrm{mg}$ tensoativo/L solução, a quantidade adsorvida é sempre maior para esta concentração de sal, aproximadamente $670 \mathrm{mg}$ tensoativo/ g rocha, do que para as outras duas concentrações estudadas. Isto ocorre devido à redução da repulsão eletrostática existente entre as espécies de tensoativo adsorvidas, o que resulta num aumento da capacidade de adsorção deste na rocha.

Pelas Figuras 1 e 2, observa-se que o equilíbrio da adsorção para a solução de tensoativo com $2 \% \mathrm{KCl}$ (Figura 1) foi atingido a uma concentração menor, $360 \mathrm{mg}$ tensativo/g rocha, que a solução de tensoativo com $2 \% \mathrm{NaCl}, 500 \mathrm{mg}$ tensativo/g rocha (Figura 2). Com isto, verifica-se, também, que para uma mesma concentração de equilíbrio, igual a $600 \mathrm{mg}$ tensoativo/L solução, a quantidade de tensoativo adsorvida na rocha de $2 \% \mathrm{KCl}$ foi menor, com $360 \mathrm{mg}$ tensativo/g rocha, do que a solução de $2 \% \mathrm{NaCl}$, com $500 \mathrm{mg}$ tensativo/g rocha, devido, provavelmente ao maior tamanho da molécula de potássio $\left(\mathrm{K}^{+}\right)$, com relação ao raio atômico, do que a do sódio $\left(\mathrm{Na}^{2+}\right)$ e a eletronegatividade do potássio ser menor que a do sódio, sendo portanto menos solúvel em água.

\subsection{Análise Termogravimétrica (TG)}

A Figura 3 mostra a perda de massa em (\%) para o sabão base puro e para o arenito adsorvido com sabão base com $4 \%$ em peso de $\mathrm{NaCl}$.

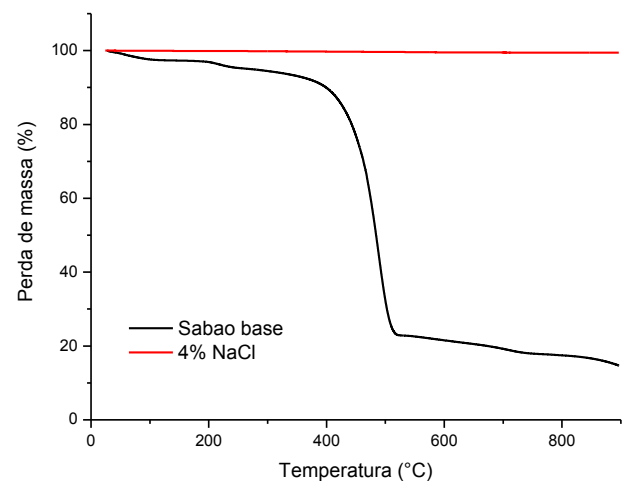

Figura 3 - Perda de massa em (\%) para o sabão base puro e para o arenito adsorvido com sabão base com $4 \%$ em peso de $\mathrm{NaCl}$, da temperatura ambiente à $900^{\circ} \mathrm{C}$, sob atmosfera de $\mathrm{N}_{2}$ e taxa de aquecimento de $10^{\circ} \mathrm{C} \mathrm{min}^{-1}$. 
Observa-se pela Figura 3 que o sabão base apresentou uma perda de massa de, aproximadamente, $70 \%$ na faixa de temperatura de $327-527{ }^{\circ} \mathrm{C}$, associada à decomposição de compostos orgânicos presentes neste tensoativo. $\mathrm{Na}$ amostra de arenito com $4 \%$ em peso de $\mathrm{NaCl}$ não foi possível observar nenhum evento térmico significativo, se mostrando estável termicamente durante toda análise da temperatura ambiente até $900^{\circ} \mathrm{C}$, havendo apenas uma pequena degradação no intervalo de temperatura de $450-500{ }^{\circ} \mathrm{C}$, provavelmente correspondente a perda de massa da quantidade de sabão base adsorvido.

\subsection{FTIR}

As Figuras 4 (a), (b) e (c) mostram o espectro FTIR para o sabão base puro, arenito puro e arenito adsorvido com tensoativo e sal, respectivamente.

O uso principal desta técnica foi detectar a estrutura de certas espécies químicas e fornecer medidas qualitativas baseadas na absorção e picos vibracionais.

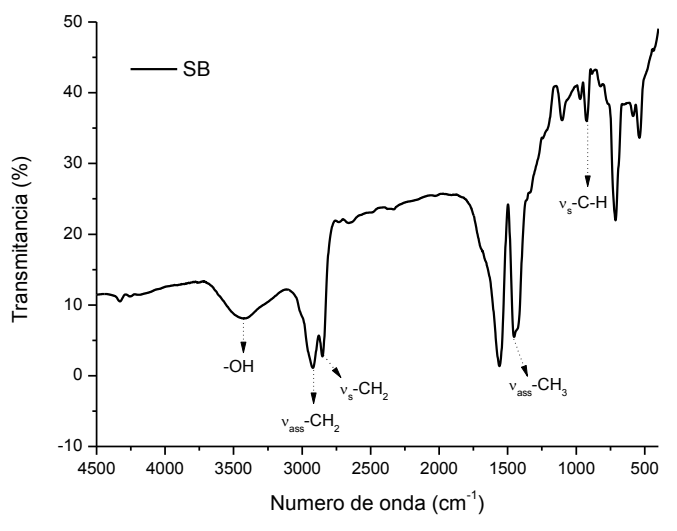

(a)

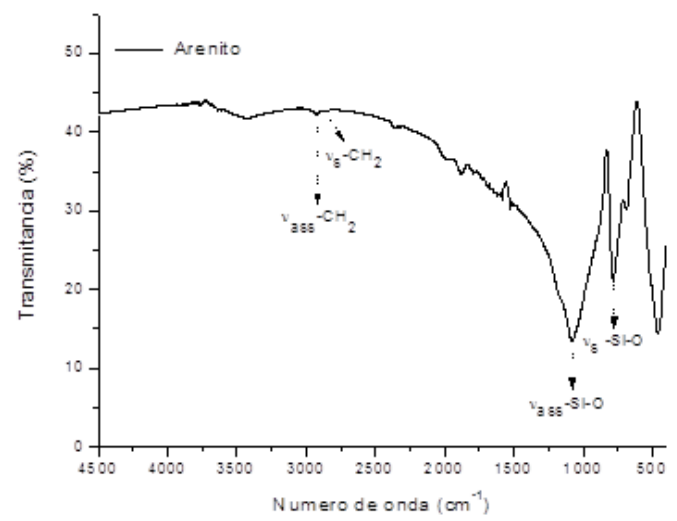

(b)

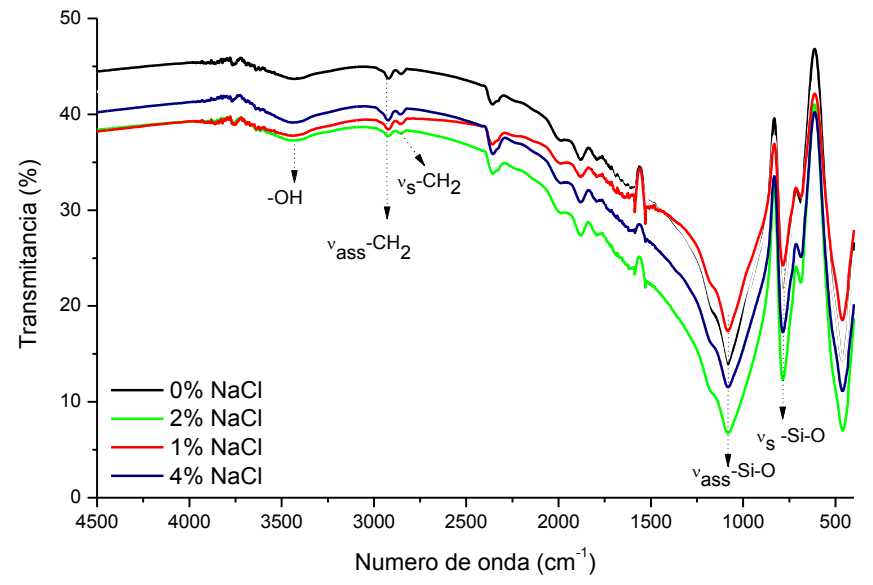

(c)

Figura 4 - Espectros FTIR para (a) sabão base, (b) arenito puro e (c) arenito adsorvido com tensoativo com várias concentrações de sal. 


\section{9 a 22 de outubro de 2014 \\ Florianópolis/SC}

O espectro FTIR do sabão base mostra picos de absorção a $916 \mathrm{~cm}^{-1}$ correspondente a deformação angular simétrica do $\mathrm{C}-\mathrm{H}$, a $1446 \mathrm{~cm}^{-1}$ correspondente à deformação angular assimétrica do $-\mathrm{CH}_{3}$ e a $2844 \mathrm{~cm}^{-1}$ e $2927 \mathrm{~cm}^{-1}$, correspondente à deformação axial simétrica e assimétrica do $-\mathrm{CH}_{2}$, respectivamente. Também pode ser visto um pico a $3438 \mathrm{~cm}^{-1}$ correspondendo à deformação axial da ligação $\mathrm{OH}$ (associado). $\mathrm{O}$ espectro FTIR do arenito puro mostra picos a $785 \mathrm{~cm}^{-1}$ e $1081 \mathrm{~cm}^{-1}$, na região de estiramento vibracional para o grupo Si-O simétrico e assimétrico, respectivamente, mostrando que o arenito contém sílica pura na sua composição principal. A amostra de arenito também mostra picos a $2844 \mathrm{~cm}^{-1}$ e $2927 \mathrm{~cm}^{-1}$, que indicam o estiramento simétrico e assimétrico do $-\mathrm{CH}_{2}$.

Em todos os casos, após a adsorção do tensoativo (Figura 4.c), bandas a $3438 \mathrm{~cm}^{-1}$ foram encontradas, originadas pelo estiramento vibracional do grupo $\mathrm{OH}$ das moléculas de água durante a adsorção do tensoativo.

\section{CONCLUSÕES}

Deste estudo, é possível verificar que as isotermas de adsorção exibiram uma adsorção em monocamada para as soluções do tensoativo aniônico (SB) com $2 \%$ em peso $\mathrm{KCl}$ e 1,2 e $4 \%$ em peso de $\mathrm{NaCl}$. A solução de tensoativo com $4 \%$ em peso de $\mathrm{NaCl}$ mostrou uma capacidade de adsorção maior do que as outras concentrações salinas, pois o sal contribuiu para a redução da repulsão eletrostática entre as espécies de tensoativo adsorvidas, aumentando a capacidade de adsorção no arenito. Portanto, para processos de EOR em que se pretende injetar uma solução do tensoativo sabão base, este mostrou ser bastante resistente a altas temperaturas podendo ser utilizados sem perder suas propriedades. Além disso, não haverá redução da eficiência do processo em razão da perda de massa por adsorção nas rochas-reservatório e da presença de sal.

\section{REFERÊNCIAS}

Curbelo, F. D. S. Recuperação avançada de petróleo utilizando tensoativos. 2006. 190 f. Tese de doutorado em Engenharia Química, Universidade Federal do Rio Grande do Norte, Natal, 2006.

Schramm, L. L. Emulsions, Fundamentals and Applications in the Petroleum Industry; American Chemical Society, Washington, DC, 1992.

Schramm, L. L. Surfactants: Fundamentals and Applications in the Petroleum Industry, Cambridge University Press, United Kingdom, 2000.

ShamsiJazeyi, H.; Verduzco, R.; Hirasaki, G. Reducing adsorption of anionic surfactante for enhanced oil recovery: Part II. Applied aspects. Colloids and surfaces A: physicochemical and engineering aspects, in press, 2014.

Somasundaran, P.; Zhang, L. Adsorption of surfactants on minerals for wettability control in improved oil recovery processes. Journal of Petroleum Science and Engineering. v.52, p.198212, 2006.

Foust, A. S.; Wenzel, L. A.; Clump, C. W.; Maus, L.; Andersen, L. B., Princípios das Operações Unitárias, $2^{\circ}$ Edição, Guanabara Dois, Rio de Janeiro, RJ, 1982. 